\title{
Why and how informatics and applied computing can still create structural changes and competitive advantage
}

\author{
Sarandis Mitropoulos \\ Regional Development, Ionian University, Lefkada, Greece, and \\ Christos Douligeris \\ Informatics, University of Piraeus, Piraeus, Greece
}

Informatics

and applied

computing

Received 7 June 2021

Revised 19 July 2021

16 September 2021

\begin{abstract}
Purpose - In the new digital age, enterprises are facing an increasing global competition. In this paper, we first examine how Information Technology (IT) can play an important role in giving significant competitive advantage in the modern enterprises. The business value of IT is examined, as well as the limitations and the trade-offs that its applicability faces. Next, we present the basic principles for a successful IT strategy, considering the development of a long-term IT renovation plan, the strategic alignment of IT with the business strategy, and the adoption of an integrated, distributed, and interoperable IT platform. Finally, we examine how a highly functional and efficient IT organization can be developed.

Design/methodology/approach - Our methodological approach was based to the answers of the following questions: 1. Does IT still matter? 2. What is the business value created by IT along with the corresponding limitations and trade-offs? 3. How could a successful IT Strategy be build up? 4. How could an effective? T planning aligned with the business strategy be build up? 5 . How could a homogenized and distributed corporate IT platform be developed? and finally, 6 . How could a high-performance IT-enabled enterprise be build up?

Findings - The enterprises in order to succeed in the new digital area need to: 1 . synchronize their IT strategy with their business strategy, 2. formulate a long-term IT strategy, 3. adopt IT systems and solutions that are implemented with elasticity, interoperability, distribution, and service-orientation. 4. keep a strategic direction towards the creation of an exceptional organization based on IT.

Originality/value - This paper is original with respect to the integrated approach the overall problem is examined. There is a prototype combined investigation of all perspectives for an effective enforcement of IT in a way that causes acceleration in competitive advantage when conducting business.
\end{abstract}

Keywords New technologies, IT strategy, Strategic alignment, Business value, SOA

Paper type Research paper

\section{Introduction}

The steadily increasing global market competition is significantly reducing the turnover time of innovation products. Information Technology (IT) can provide a significant competitive market advantage to an enterprise because it dynamically promotes relationships with its customers and suppliers, strengthens strategic alliances, promotes innovative products and services, adapts existing solutions, and achieves lower operating costs, while it improves the internal business processes. It is true that new technologies, such as the cloud, mobile computing, the Internet of Things (IoT), and the use of artificial intelligence (AI) have brought

(C) Sarandis Mitropoulos and Christos Douligeris. Published in Applied Computing and Informatics. Published by Emerald Publishing Limited. This article is published under the Creative Commons Attribution (CCBY 4.0) licence. Anyone may reproduce, distribute, translate and create derivative works of this article (for both commercial and non-commercial purposes), subject to full attribution to the original publication and authors. The full terms of this licence may be seen at http://creativecommons. org/licences/by/4.0/legalcode

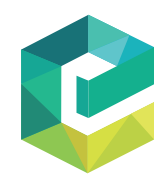

Applied Computing and Informatics Emerald Publishing Limite e-ISSN: 2210-8327 p-ISSN: 2634-1964 DOI 10.1108/ACI-06-2021-0149 
around structural changes in the way businesses operate as well as in the way markets function. For example, the internet has created hyperarchies that influence the creation of new business models [1], by replacing the traditional enterprise hierarchies, while on the other hand it has necessitated a comprehensive review of the traditional business strategies. This revision of the companies' business strategies, as well as the new supply value chain have significantly increased the productivity, the quality of services offered to customers and the overall value proposition of the enterprises.

Nevertheless, there are many enterprises, which, despite investing a significant amount of money and human resources in applied computing, as well as in relevant research, have failed in having the initially expected advantages. This happened because these enterprises failed to adopt and then successfully implement the new e-business and e-governance models [2]. In the context of rapidly responding to market needs and to their fierce competition, many enterprises have only pursued short-term benefits from applied computing and informatics by trying only to accelerate the development of new services and products at the lowest possible operating costs. But ignoring the fundamentals of strategy formulation has led to a convergence of business practices based on cost leadership [3], something we have seen happening in the past with the dotcom enterprises. This problem still exists with the latest informatics technologies, where we see that enterprises still do not incorporate the informatics and computing applications in their processes in the right way $[2,4,5]$. The main faults that enterprises make regarding the adoption of informatics are that they do not:

(1) synchronize their IT strategy with the goals of their business strategy,

(2) formulate a long-term IT strategy,

(3) keep a strategic direction towards the creation of an exceptional organization based on IT,

(4) adopt effective IT systems, and integrated IT solutions; instead these are implemented hastily, inelastically and without interoperability.

The exponential growth of ubiquitous computing drives the need for new business models, which must serve an effective IT-enabled business strategy directly related to the Digital Transformation. The problem space here is how the effective adoption of new IT technologies can be achieved which in turn drives the requirements of renewed business strategy and processes, and of culture change [29]. The main problem which modern enterprises face concerns the right IT implementation along with the adaptation of the right strategy, process, and culture. This paper tries to answer all these requirements in a consistent and methodological way. Even though a considerable body of research towards this direction exists, there is still a gap for the development of a perfect alignment between the strategy the enterprises must follow and an intelligent and effective way the new technologies are implemented so that they create a competitive advantage [5]. Internet, cloud computing, mobile computing, software-oriented architectures (SOA), Internet of things (IoT), blockchain, server virtualization and other modern technologies provide the enterprises with the opportunity to migrate from the traditional business models to new ones [30, 31, 35, 36].

Although there exist theoretical frameworks in the literature [2-5], there is still a lack of of frameworks that combine the characteristics of hybrid, both research and practical methods approaches. The outcomes of the existing approaches show that the use of IT has not been proven fully effective, except from some exceptional cases.

To achieve an effective IT incorporation, this paper proposes a framework with very specific suggestions. This framework can be used as a roadmap for future readiness and growth. The evaluation discussion shows the effectiveness of the proposed framework and methodology. This framework in short consists of the following steps: 
(1) Examination of the expected business value to be created by IT along with any expected limitations and necessary trade-offs.

(2) Formulation of an effective IT strategy aligned with the overall business strategy.

(3) Development of an effective IT planning.

(4) Development of a homogenized, interoperable, and distributed IT platform.

(5) Development of a high IT-enabled organization in all its dimensions.

(6) Establishment of an on-going evaluation system for the continuous improvement of the IT strategy and the corresponding infrastructure.

The issues and steps mentioned above are thoroughly analysed and potential solutions are proposed. A thorough and comprehensive investigation of whether IT can still make structural changes in the operation of businesses is performed. In addition, it is examined how this can be achieved with respect to the creation of business value considering the corresponding limitations and necessary trade-offs. But since creating value without developing a rational strategy is impossible the principles of a sound IT strategy are examined, as well as how this can be effectively developed. Finally, implementation issues and evaluation results are discussed, as well as potential future work.

\section{IT still does matter}

Many researchers argue that IT no longer offers any innovation over the competition and have, therefore, IT has reached the stage of maturing as a service [6]. This viewpoint has been expressed without considering its high and disruptive evolution. There is obviously a part of IT that is common to almost all enterprises, making this utility approach workable. Nevertheless, this is only one side of the coin because IT is creating new situations that accelerate the developments in the operation of markets and enterprises. The concept of ubiquitous computing, for example, is a new trend which will surely cause structural changes, while new ideas in virtual communities can create significant changes in corporate collaboration by forming on-demand virtual business partner hyperarchies. In addition, new intelligent algorithms for machine learning, artificial intelligence and biotechnology are being developed, thus facilitating research into new products (e.g. drugs and crystal solid materials) and services (e.g. telemedicine) with significant business and social benefits [7]. Thus, the new information technologies can leverage the capacity for innovation. Enterprises through the smart and targeted use of IT will be able to create growth, and innovative products and services at the right time.

In addition, enterprises need to focus on another perspective of IT, that of the growing business value through digital culture. A well-designed business strategy, synchronized with that of IT, creates an innovation-oriented corporate culture. Such a culture is not easy to be copied from the competition, thus, giving the enterprises that adopt it a clear lead. There are many important examples that prove this to be true, such as the ones of Toyota and Dell - their supply chain and production chain management practices make them stand out [6].

The innovative and smart use of IT (e.g. green informatics) can create an additional competitive advantage which, most of the times, is closely connected with a change of culture [8]. However, the technology is not a panacea. In practice, many enterprises do not effectively enforce the new IT-enabled business models because their adoption of IT has not been directed to develop a highly IT-enabled corporate culture, they have not integrated their digital strategy with the overall business strategy, or they have not adequately understood the new technology trends while developing a corresponding strategy. 


\section{Business value creation: limitations and trade-offs}

Nowadays, the relationship between business strategy and IT-based innovation is a strongly interacting one. A successful business strategy must define an IT infrastructure driven by innovation. Obviously, all of the expected benefits offer improved business performance and are, therefore, critical to the business success $[5,9]$.

IT enables an increased control of the operating costs as well as of productivity. Indeed, the assembly of products e.g. through the Computer Integrated Manufacturing (CIM) and the Robotics systems of the industry 4.0 era, increases productivity and quality, while reducing costs. We also notice that information technology significantly increases the knowledge of the business environment through monitoring systems, such as the ones used in the product delivery status or in the stock levels of warehouses. IT systems can help develop staff skills through e-learning and on-the-job on-line assistance.

Nevertheless, there exist several criteria which need to be fulfilled for enterprises to be able to differentiate from competitors. Such criteria include among others the brand name visibility, the quick-to-market response, and the service quality [10]. Despite these advantages, there are important limitations that need to be considered, such as the existence of isolated and proprietary software applications and databases. Outdated information (legacy) systems, which require high-cost adapters to bridge the newer applications, and the use of a wide range of different and heterogeneous technologies, are two other examples. The low utilization of the existing IT resources is also a problem. All these limitations significantly reduce the efficiency of IT, the process support, the access and dissemination of information, the implementation of new projects, and the staff adaptation to the new conditions, while they further increase the operating costs.

There are solutions to such problems, which enterprises are called upon to adopt, such as e.g. the Enterprise Application Integration (EAI), the rapid development of new applications, and the development of business process management systems By gradually adopting such solutions an enterprise can in a reasonable time develop a much more integrated business environment, launch new services in a timely fashion, achieve world-wide access, improve its business operations control, acquire resources on demand, work on a flexible infrastructure and lower costs $[2,10]$.

Most of the times, the enforcement of these IT solutions requires the consideration of a variety of trade-offs, while the main question is: "is there adequate capacity, qualities and strategic direction for a change from the old economy environment to the new one?". The answer to this question includes: (1) the adoption of an innovation culture, which in turn requires management of change and capability building up for the human resources, and (2) an elastic and interoperable infrastructure $[11,12]$.

In addition, the changing of an operational business model can drive the requirements for higher adaptation in the application software portfolio creating another trade-off among efficiency, innovation, experimentation, and conformance to the relevant standards [11-13].

\section{The proposed methodological approach}

Given that IT is still considered to be able to bring about significant structural changes and provide a business competitive advantage in the modern markets along with all the related operational benefits, the question is how this goal can be achieved methodologically. This development can be considered to have the following four main dimensions:

(1) Development of a successful IT strategy, whose goals should be synchronized with the business strategy, e.g. there can be no strategy to penetrate global markets, while the company's information system cannot support the internationalization of the information it offers and manages. 
(2) The IT development plan which should consider the organizational structure of enterprise, the demanded quality of service to its customers, the firm's human resources and the existing IT infrastructure and systems. Everything may need to be changed partially or to a very large extent.

(3) Development of the IT Infrastructure as a homogenized and distributed service platform provided either to the internal customers of the company or to the external ones.

(4) Development of services and policies for management, research and innovation, training for the creation of a technologically high-performance enterprise,

All these key dimensions of development of innovation and IT solutions are shown in Figure 1 as phases.

\section{Building up a successful IT strategy}

A business strategy expresses the vision, the mission and the main business goals of a enterprise or organization. The business goals must be interpreted into subgoals that are enforced on specific high-level business domains, e.g. sales, customer relationships, production, and logistics. In fact, these domains, according to the balanced scorecard (BSC) approach [5], are influenced by the corporate strategy regarding the perspectives of the future readiness and the innovation, the internal process improvement, the customer orientation, the cost control, and the financial goals. It is obvious that enterprises need to effectively approach these perspectives if they want to achieve a transition from the oldfashion business models to the new ones. Thus, the strategic components incorporated in these perspectives, must be refined and improved.

These components, in fact, construct the high-level business domains upon which the goals of the business strategy must be enforced. Of course, these domains must contain subdomains or, in other words, lower-level domains that express the implementation components of an enterprise or organization. Such components include among others technical processes, business data, operations, and informatics implementations. This topdown approach is very useful for the executives because it provides them with a tool for the successful enforcement of a business strategy considering all its dimensions.

The IT potentiality in terms of new technologies and solutions, makes the IT strategy to be the determinant while the business strategy to be the weak area which needs improvement [5]. This fact is expressed by the Strategic Alignment (SA) model [14] for the business and IT strategies and the organisational and IT infrastructures.

For example, ubiquitous computing brings new capabilities to the enterprises and, thus, it pushes for a revision of the corresponding business strategies. The goal is to achieve a

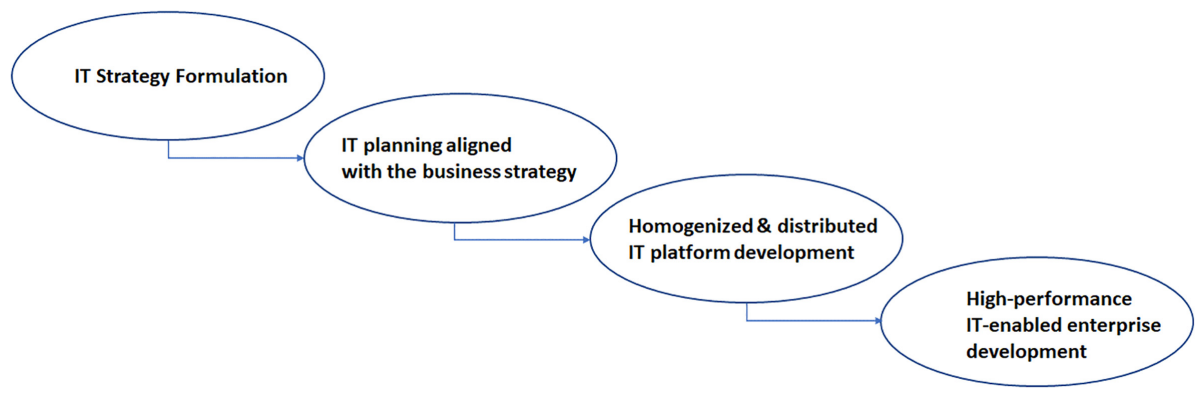

Figure 1. The basic dimensions of a high-performance enterprise based on innovation and new technologies

Informatics and applied computing 
competitive advantage over the competition through the development of new products and the appropriate modification of the business scope, the distinct competencies, and the business governance, along with the improvement of the organizational infrastructure which concerns the business processes, the human resource capability, and the administrative structure. As mentioned, this approach helps enterprises to conform to the new requirements that arise due to the new technological solutions and the potentialities created by them. Towards this direction, the following are proposed:

(1) IT and business strategy alignment,

(2) dimensioning of IT resource requirements,

(3) adoption of new IT architectures and their management, based on innovation and adaptability, and

(4) selection of Key Performance Indicators (KPI) for evaluation reasons.

The first point imposes an IT strategic plan fully aligned with the business strategy. The next point asks for an open, interoperable, scalable, and distributed IT infrastructure, while the third point relates to a high-performance IT-enabled enterprise [4, 5, 15]. Key performance metrics are always required so that the evaluation of the adopted overall approach to be possible.

\section{Developing an effective IT planning aligned with the business strategy}

IT planning must incorporate the current as well as the future enterprise needs and technological trends. Thus, it must cover long-term issues as well as whatever is necessary for future readiness. A Strategic Alignment (SA) between the business and IT strategies addresses four main areas: business strategy, IT strategy, organizational infrastructure, and IT infrastructure $[5,16]$. These areas need to be aligned with each other according to the business requirements and the type of enterprises. Namely, the requirement for alignment varies from enterprise to enterprise and while it is generally very helpful, it may, however, limit the degrees of freedom in some business cases. In fact, trade-offs and equilibria between strategic alignment and flexibility must be addressed successfully. For example, the production and other business operations, like the customer relationship management (CRM), are positively affected by the Strategic Alignment. On the other hand, the business planning, the marketing, and the sales are less influenced by the SA. Specifically, enterprises, whose critical operations do not focus on IT, do not require such a strict SA. The more supportive and functional the role of IT is, the more SA it requires. On the contrary, where IT moves within a strategic role, the need for a strict SA decreases [17-19]. The following perspectives can be considered in an IT environment [20]:

(1) IT infrastructure: emerging IT infrastructure technologies and solutions call for the reformulation of the IT strategy, while the business strategy is implicitly impacted.

(2) IT organization infrastructure: emerging IT calls for the reformulation of the organizational infrastructure, while the business strategy is implicitly impacted.

(3) competitive potentiality: emerging IT capabilities, as well as new IT governance patterns call for the reformulation of the business strategy, while the organizational infrastructure is implicitly impacted.

(4) service level: the IT resources use, as well as the orientation to the customer calls for the reformulation of the IT infrastructure, while the organizational infrastructure is implicitly impacted. 
7. Developing a homogenized and distributed corporate IT platform

The following services can be offered in an enterprise network in an open and interoperable manner [21, 22]:

(1) electronic services for channel-management, as far as all the involved parties, like enterprises, clients, and suppliers, are concerned,

(2) security, that concerns the IT resources protection,

(3) communication mechanisms needed for the communication and interworking between all the internal/external business entities,

(4) database and file management services, for the purpose of making the required data and files available over the enterprise network,

(5) application services, for the purpose of making the required applications, like EPR, SCM and HRM, available over the enterprise network, and

(6) management of IT facilities, needed for integration and synchronization of the infrastructure layers and for provision of servers and platforms.

New trends in IT technologies call for the distribution, homogenization, integration, and interoperability of systems and services [23]. Open IT standards and SOA are among the current information systems technologies that enterprises need to move towards because they offer increased interoperability, transfer of application services to heterogeneous environments, enterprise application integration, service reusability, high operational control and monitoring, and flexible service configuration and measurability in service performance [24].

A SOA helps to create networks of services for the purpose of their common management. Service orientation here is mentioned as an architectural approach of the new IT systems without restricting its implementation to specific offered solutions, as it is the Enterprise Service Bus (ESB). In most cases, service orientation must be followed due to the benefits mentioned above. Figure 2 introduces the conceptual approach of a service grid, where application services are interacting each other through several underlying platform services, like routing, message exchange and message queuing, data and knowledge management, distributed API's, security, accounting, QoS assurance, system monitoring and control.

Indeed, the adoption of IT service grids can prove to be a key factor for enterprises in their efforts to gain a significant competitive advantage. New informatics and applied services need to be distributed, scalable, open, and reliable in a low cost and quick-to-market

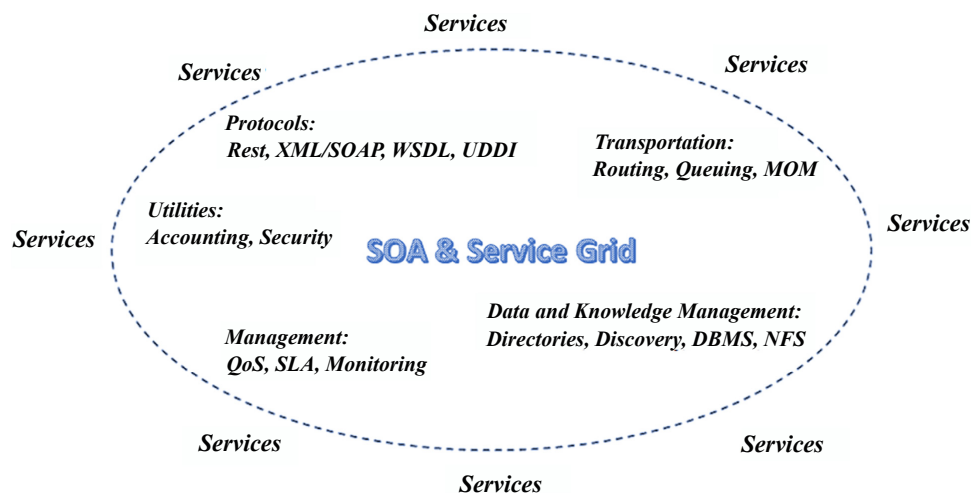

Informatics and applied computing 
deployment fashion. Furthermore, this adoption will cause channel enhancements, like disintermediation, mitigation of information asymmetry, world-wide access, virtualization, cost reduction and control, efficient management information, economies of scale, and improved strategic positioning.

Service grids will be enhanced with the new ubiquitous computing capabilities. For example, mobile computing can effectively leverage the quality of service, as well as the collaboration between employers, employees, customers, strategic partners, and third parties [25]. Furthermore, smartphones can provide a variety of functionalities, like user interaction, task management, user online help, blogging, wiki, chatting, and remote access from trusteed parties or customers through appropriate authentication and authorizations, that can significantly leverage the operational effectiveness of modern enterprises. The dissemination of notifications (e.g. Google Cloud Messages) in mobile apps is another example which can facilitate business operations.

\section{Developing a high-performance IT-enabled enterprise}

The IT services implemented with technologies like SOA and ubiquitous computing, must be supplemented by IT management services. Towards this direction [21, 24], the following management services can be identified:

(1) IT administration services, for the platforms, the IT systems planning and the project management, the SLAs, and the negotiations with the IT suppliers,

(2) IT architecture services, which need the incorporation of system management policies for the effective management of IT resources,

(3) IT R\&D services, concerning new products, services, processes, and operations using IT, and

(4) training services in the use of IT, strongly needed for the capacity building of enterprise's staff.

Along with the management, the overall business processes as well as any activity involved in a process need to be designed effectively. It has been well-documented that business processes which are supported by IT, along with the right organizational and management services, are significantly more efficient than those of the old economy [26, 27]. The supply chain is a classic example that proves the truth of the matter. Figure 3 illustrates the process of supply chain. In brief, creating a performance-oriented IT organization requires process innovation and efficiency, effective communication with the customers, the partners, and the suppliers, inventory management, development of new products and services, and agile adaptation of the existing ones.

IT-enabled enterprises need to be supported by several key functionalities and technologies [22, 28, 33, 34]. Bridges between the different information systems via appropriate interfaces by using e.g. web services (XML or json-based approaches), are also required. Of course, the adoption of these technologies may raise critical security and risk management issues., All the vulnerabilities and security holes must be eliminated in an way.

Figure 3.

The supply chain

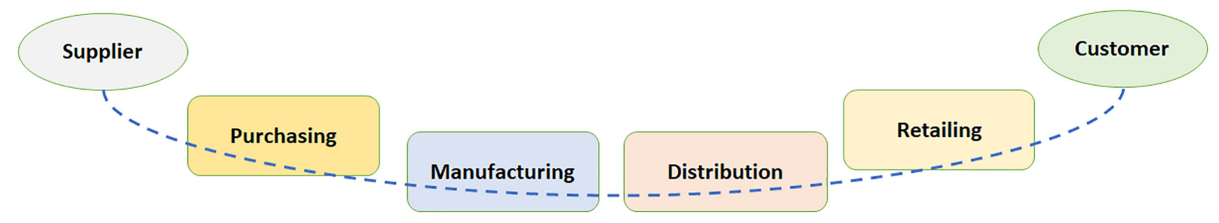




\section{Analysis discussion and evaluation}

Hereafter, some examples are provided regarding the right way to employ new technologies in modern enterprises. As mentioned in the Accenture report "Improving Business ROI with Digital Technologies"[1], "to become a more efficient finance organization, considerable investments must be made to improve processes and technology". According to this survey, the reasons of fail are that (1) there is no clear strategy and vision, (2) they do not deal effectively with the legacy systems, and (3) they do not understand adequately the existing digital capabilities. The outcomes of this survey are fully aligned with the assumptions of our research presented above.

In the report entitled "The ROI of IoT: The 7 benefits it can bring to your business"[2], it is stated that "if you know how your key equipment and assets are behaving, and how people are interacting with them, you can unlock the value of that data through an Io T software program that delivers actionable insights and improves business processes in future". This is a key issue for the development of an improved long-term planning and strategy, which in turn it is necessary for the success of an IT investment project, according to our research.

According to [32], 88\% of companies reported that they already use cloud services, while $50 \%$ of companies expect to have all their data stored in the cloud for a period of two years. The same survey revealed that most enterprises already have applications in production based on cloud at a level of about $47 \%$, while a significant percentage intend to develop such applications. However, these enterprises are almost entirely concerned with the security of their data, which requires a high-performance IT-enabled enterprise according to our research.

Finally, according to the study conducted in [22], by adopting virtualization technology for a computer centre, the cost of investing in it gradually yielded positive profits over the annual investment cost over a period of five years. Also, the return on investment (ROI) became positive after the second year, while during the fourth year, the total investment cost became significantly lower than the projected profits.

It should be noted that for a continuous evaluation of the IT strategy and its implementation, it is necessary to define critical success factors of the organizational and the IT infrastructures that are linked to certain KPIs. These indicators will assess not only the effectiveness of internal processes, customer satisfaction or financial gains, but also the achievement of the structural changes necessary to adapt the organization to an ever-evolving environment.

\section{Conclusions and future work}

This paper presented in a concise and detailed way, the very important role that IT still plays nowadays in modern enterprises, giving them a competitive advantage in modern globalized markets. It was posited that enterprises that adopt IT in a smart and innovative way have a significant capacity building capability, improved future readiness, and better strategic positioning.

In the future, we intend to evaluate our approach in an enterprise. The process of analysis will include the business strategy, processes and practices, organizational structures, IT infrastructure, etc. The whole task faces the restrictions of the confidentiality of business information making access to all this information rather difficult, especially when this information may be made public. An organization before the application of our methodology requires an adequate mapping (a master plan), while then a road map should be developed with all the technical details of integration of practices, plans, technological solutions, evaluations, etc. Evaluations could be based among others in the implementation of a balanced scorecard, where the results will be expressed via Key Performance Indicators concerning crucial success factors (CSF) in all, the internal processes, the quality of services to the customers, and the financial profit which is essentially the main motivation of every enterprise.
Informatics and applied computing 


\section{Notes}

1. https:/www.accenture.com/nl-en/blogs/insights/how-digital-technologies-improve-business-roi (last access: 14/7/2021).

2. https://blog.worldsensing.com/critical-infrastructure/roi-iot/ (last access 14/7/2021).

\section{References}

1. Naved K, Tabassum F. Reinventing business organizations: the information culture framework. Singapore Management Rev. 2005; 27(2): 37-63.

2. Porter ME, Heppelmann JE. How smart, connected products are transforming competition. Harv Business Rev Spotlight Managing Internet Things. 2014, November 2014.

3. Teece DJ. A capability theory of the firm: an economics and (strategic) management perspective. Taylor \& Francis Online. 2017, New Zealand Economic Papers; 53(1) 2019.

4. Tiwana A. IT strategy for non-IT managers. MIT Press. 2017, London, England.

5. Mitropoulos S. An integrated model for formulation, alignment, execution and evaluation of business and IT strategies. Int J Business Syst Res. 2021; 15(1): 90.

6. Vandenbosch B, Lyytinen K. Much ado about IT: a response to "the corrosion of IT advantage" by Carr' NG, J Business Strategy. 2004; 25(6): 10-12.

7. Schmidt J, Marques MRG, Botti S, Marques MAL. 2019. Recent advances and applications of machine learning in solid-state materials science. Available at: https://www.nature.com/articles/ s41524-019-0221-0.

8. Benlamri R, Sparer M. Leadership, innovation and Entrepreneurship as driving forces of the global economy. Proceedings of the 2016 ICLIE, Springer Proceedings in Business and Economics. 2016.

9. Koi-Akrofi GW. Justification for IT investments: evaluation methods, frameworks, and models. Texila Int J Management. 2017; 3(2).

10. Melarkode A, From-Poulsen M and Warnakulasuriya S. Delivery agility through IT. Business Strategy Rev. 2004, Autumn 2004.

11. Wadhwa M, Harper A. Technology, innovation and enterprise transformation. IGI Glob book Ser Adv Business Inf Syst Analytics (Abisa). 2015, Hershey, USA.

12. Schrage M, Kiron D, Hancock B, Breschi R. Performance management's digital shift. MIT Sloan Management Rev. February 26, 2019. Available at: https:/sloanreview.mit.edu/projects/ performance-managements-digital-shift/.

13. Dong J and Yang $\mathrm{CH}$. Business value of big data analytics: a systems-theoretic approach and empirical test. Inf Management. 2020; 57(1): 103124.

14. Ilmudeen, A, Bao, Y, Alharbi, IM. How does strategic alignment affect firm performance? the roles of information technology investment and environmental uncertainty, J Enterprise Inf Management. 2019; 32(3): 457-76.

15. Davies P. Strategic objectives and principles. 2015. Version V1.0, University of Sussex, Date of Issue: 9th April 2015 Available at: http://www.sussex.ac.uk/its/pdfs/IT_Strategy_2015.pdf.

16. Chugunov A, Misnikov Y, Roshchin E, Trutnev D. Electronic governance and open society: challenges in Eurasia. 5th International Conference. 2018, EGOSE 2018.

17. Tallon, P. How information technology infrastructure flexibility shapes strategic alignment: a case study investigation with implications for strategic IS planning. Plann Inf Syst. 2015; 15, Acemap: 425-55.

18. Tallon P, Kraemer K. Investigating the relationship between strategic alignment and IT business value: the discovery of a paradox, In: Shin N (Eds), Creating business value with IT: challenges and solutions, Hershey, Pennsylvania, PA: Idea Group Publishing, 2003.

19. Kaleka A, Morgan NA. How marketing capabilities and current performance drive strategic intentions in international markets. Ind Marketing Management. 2017; 78: 108-121. 
20. Coleman P, Papp R. Strategic alignment: analysis of perspectives. Proceedings of the 2006 Southern Association for Information Systems Conference, March 11-12, 2006, Florida, FL. 2006.

21. Weill P, Subramani M, Broadbent M. Building IT infrastructure for strategic agility. MIT Sloan Management Rev. 2002, Fall 2002.

22. Lambropoulos G., Mitropoulos S., Douligeris C. Improving business performance by employing virtualization technology: a case study in the financial sector. Computers. 2021; 10(4): 52.

23. Razis M., Mitropoulos S. An integrated approach for the banking intranet/extranet information systems: the interoperability case. Publ Int J Business Syst Res. 2021, Inderscience Publishers. forthcoming paper.

24. Katsikogiannis G., Kallergis D., Garofalaki Z., Mitropoulos S., Douligeris C. A policy-aware service oriented architecture for secure machine-to-machine communications. J Ad Hoc Networks. 2018; 80, November 2018, Elsevier Science Publishers.

25. Chassapis P, Mitropoulos S, Douligeris C. A prototype mobile application for the athens numismatic museum. J Appl Comput Inform. 2020; ahead-of-print(ahead-of-print), Emerald Publishers.

26. Mitropoulos S. A simulation-based approach for IT and business strategy alignment and evaluation. Int J Business Inf Syst. 2012; 10(4): 369-396, Inderscience Publishers.

27. Mitropoulos S, Giannakos K, Achlioptas J, Douligeris C. A prototype workflow MIS for supply chain management: architecture, implementation and business evaluation. Publ Int J Business Inf Syst. 2021, Inderscience Publishers. forthcoming paper.

28. Mitropoulos S, Mitsis C, Valacheas P, Douligeris C. An online Emergency medical management information system using mobile computing. J Appl Comput Inform. 2020. online version, Emerald Publishers.

29. Sayabek, Z, Suieubayeva, S, Utegenova, A. Digital transformation in business, ISCDTE 2019: digital age: chances, challenges and future lecture notes in networks and systems. 2020; 84: 408-415, Springer, Cham.

30. Gimpel H, Röglinger M. Digital Transformation Changes and chances- Insights based on an empirical study. 2015. Available at: https://fim-rc.de/wp-content/uploads/2020/02/FraunhoferStudie_Digitale-Transformation.pdf.

31. IDC. Exploring the Impact of infrastructure virtualization on digital transformation strategies and carbon emissions, white paper. 2019, Available at: https://www.vmware.com/content/dam/ digitalmarketing/vmware/en/pdf/company/vmware-exploring-impact-of-infrastructurevirtualization-on-digital-transformation-strategies-and-carbon-emissions-whitepaper.pdf.

32. Oracle KPMG. Oracle and KPMG cloud threat report. 2020, Available at: https://www.oracle.com/ a/ocom/docs/cloud/oracle-cloud-threat-report-2020.pdf, [Accessed 12 June 2021].

33. Huang $\mathrm{M}$, et al. An effective service-oriented networking management architecture for 5Genabled internet of things. Compuert Networks. 2020; 173: 107208.

34. Niu Y, et al. Exploiting device-to-device communications in joint scheduling of access and backhaul for mmWave small cells. IEEE JSAC. 2015; 33(10): 2052-2069.

35. Ahmad Qadri Y, et al. The future of healthcare internet of things: a survey of emerging technologies. IEEE Commun Surv Tutorials. 2020; 22(2): 1121-1167.

36. Bera B, et al. Designing blockchain-based access control protocol in IoT-enabled smart-grid system. IEEE Internet Things J. 2021; 8(7): 5744-5761.

\section{Corresponding author}

Sarandis Mitropoulos can be contacted at: smitropoulos@ionio.gr

For instructions on how to order reprints of this article, please visit our website:

www.emeraldgrouppublishing.com/licensing/reprints.htm

Or contact us for further details: permissions@emeraldinsight.com 